\title{
Dynamic Single-Pile Nim Using Multiple Bases
}

\author{
Arthur Holshouser \\ 3600 Bullard St, \\ Charlotte, NC 28208, USA \\ Harold Reiter \\ Department of Mathematics, \\ University of North Carolina Charlotte, \\ Charlotte, NC 28223, USA \\ hbreiter@email.uncc.edu
}

Submitted: Jun 25, 2004; Accepted: Mar 23, 2006; Published: Mar 30, 2006

Subject classifications: $91 \mathrm{~A} 46$

\begin{abstract}
In the game $G_{0}$ two players alternate removing positive numbers of counters from a single pile and the winner is the player who removes the last counter. On the first move of the game, the player moving first can remove a maximum of $k$ counters, $k$ being specified in advance. On each subsequent move, a player can remove a maximum of $f(n, t)$ counters where $t$ was the number of counters removed by his opponent on the preceding move and $n$ is the preceding pile size, where $f: N \times N \rightarrow N$ is an arbitrary function satisfying the condition (1): $\exists t \in N$ such that for all $n, x \in N, f(n, x)=f(n+t, x)$. This note extends our paper [5] that appeared in E-JC. We first solve the game for functions $f: N \times N \rightarrow N$ that also satisfy the condition (2): $\forall n, x \in N, f(n, x+1)-f(n, x) \geq-1$. Then we state the solution when $f: N \times N \rightarrow N$ is restricted only by condition (1) and point out that the more general proof is almost the same as the simpler proof. The solutions when $t \geq 2$ use multiple bases.
\end{abstract}

\section{Introduction}

Notation $1 N$ is the set of positive integers, and $N_{0}=\{0\} \cup N$. Let $f: N \times N \rightarrow N$ be a function satisfying the condition (1): $\exists t \in N$ such that for all $n, x \in N, f(n, x)=f(n+$ $t, x)$. If $x, y \in N_{0}$, then $\oplus$ is defined by $x \oplus y \equiv x+y(\bmod t)$ and $x \oplus y \in\{0,1,2, \ldots, t-1\}$. Thus $x \oplus y$ is uniquely specified. Note that $(\{0,1,2, \ldots, t-1\}, \oplus)$ is a cyclic group. 
The Games. Suppose game $G_{0}$ with move function $f$ is given. Then we define the games $G_{i}, i=1,2, \cdots, t-1$ where $G_{i}$ is the same as game $G_{0}$ except the move function $f(i \oplus n, x)$ is used in the place of $f(n, x)$.

Example 1 In game $G_{0}$, suppose the moving player is facing a pile size of 10 counters and the preceding pile size was 15 counters. This means his opponent removed 5 counters on the preceding move. Also, suppose $f(15,5)=5$. This means the moving player can remove from the 10 counter pile $1,2,3,4$ or 5 counters. If $f(15,5) \geq 10$, the moving player can remove all 10 counters and immediately win.

Definition 1 In game $G_{i}, i=0,1,2, \cdots, t-1, \forall n \in N, g_{i}(n)$ is defined to be the smallest winning move size for a pile of $n$ counters. Also, $g_{i}(0)=\infty$. This means that the removal of $g_{i}(n)$ counters from a pile of $n$ counters is a winning move in $G_{i}$, and $\forall t \in N$, if $1 \leq t<g_{i}(n)$ the removal of $t$ counters from a pile of $n$ is a losing move in $G_{i}$. Of course, $\forall n \in N, 1 \leq g_{i}(n) \leq n$.

Algorithm 1 Since $g_{i}(0)=\infty$ it is easy to see that $\forall n \in N, g_{i}(n)$ is the smallest $t \in\{1,2,3, \cdots, n\}$ such that $f(i \oplus n, t)<g_{i}(n-t)$. This means $g_{i}(1), g_{i}(2), \cdots$ can be computed recursively.

Definition 2. A strictly increasing sequence $B=\left(b_{0}=1, b_{1}, b_{2}, \cdots\right)$ of positive integers with $b_{0}=1$ is called a base. $B$ can be finite or infinite.

Bases for Games. Suppose we are given games $G_{i}, i=0,1,2, \cdots, t-1$, with move functions $f(i \oplus n, x)$ where $f: N \times N \rightarrow N$ satisfies (1) $\exists t \in N$ such that $\forall n, x \in N$, $f(n, x)=f(n+t, x)$ and $(2) \forall n, x \in N, f(n, x+1)-f(n, x) \geq-1$. For each $G_{i}$, $i=0,1,2, \cdots, t-1$, we assume that a base $B_{i}=\left(b_{i 0}=1, b_{i 1}, b_{i 2}, \cdots\right)$ has been generated that satisfies the following conditions.

First, $b_{i 0}=1, b_{i 1}=2, i=0,1,2, \cdots, t-1$. Also, $\forall i \in\{0,1,2, \cdots, t-1\}, \forall k \geq$ $1, b_{i, k+1}=b_{i k}+b_{i \oplus b_{i k}, j}$ where $b_{i \oplus b_{i k}, j}$ is the smallest member of $B_{i \oplus b_{i k}}$ such that

$$
(* *) f\left(i \oplus b_{i k} \oplus b_{i \oplus b_{i k}, j}, b_{i \oplus b_{i k}, j}\right) \geq b_{i k}
$$

if such a $b_{i \oplus b_{i k}, j}$ exists. Also, each base $B_{i}$ has been generated as far as possible. This means that $\forall i \in\{0,1,2, \cdots, t-1\}$, if $\left\{b_{i 0}, b_{i 1}, \cdots, b i k\right\} \subseteq B_{i}$ then $\left\{b_{i 0}, b_{i 1}, \cdots, b_{i k}, b_{i, k+1}\right\} \subseteq B_{i}$ when $\exists b_{i \oplus b_{i k}, j}$ that satisfies (**). Starting with $b_{i 0}=1, b i_{1}=2, i=0,1,2, \cdots, t-1$, we can generate $B_{0}, B_{1}, B_{2}, \cdots, B_{t-1}$ in some definite order. For example, we might add one member to $B_{0}$, if possible, add one member to $B_{1}$, if possible, add one member to $B_{2}$, if possible, $\cdots$, add one member to $B_{t-1}$, if possible. Then we repeat this cycle $B_{0}, B_{1}, B_{2}, \cdots, B_{t-1}$. We leave it to the reader to show that no matter what order $B_{0}, B_{1}$, $B_{2}, \cdots, B_{t-1}$ is generated, if we generate as far as possible then these bases will always 
be exactly the same. Also, we point out that for some $i \in\{0,1,2, \cdots, t-1\}, B_{i}$ might be infinite, and for other $i \in\{0,1,2, \ldots, t-1\}, B_{i}$ might be finite.

The following definition is very convenient in proving Theorems 1,2.

Definition 3. For each game $G_{i}, i=0,1,2, \cdots, t-1$, we define a function $F_{i}: N_{0} \times$ $N \rightarrow\{0,1\}$ as follows. First, $\forall x \in N, F_{i}(0, x)=0$. Also, $\forall n, x \in N, F_{i}(n, x)=0$ if $1 \leq x<g_{i}(n)$ and $F_{i}(n, x)=1$ if $g_{i}(n) \leq x$.

This means that if $n \in N$ is fixed and $x \in N$ is a variable then the sequence $F_{i}(n, x)$, $x=1,2,3, \cdots$ always consists of a finite string (possibly empty) of consecutive 0's followed by an infinite string of consecutive 1's. Of course, $F_{i}(n, x)=1$ when $x \geq n$. From the definition of $g_{i}$ and $F_{i}$, we note that for all $n, x \in N$ if $x \leq n$ then $F_{i}(n, x)=1$ when the list $F_{i}(n-1, f(i \oplus n, 1)), F_{i}(n-2, f(i \oplus n, 2)), \cdots, F_{i}(n-x, f(i \oplus n, x))$ contains at least one 0. Also, $F_{i}(n, x)=0$ when this list contains no 0's. Furthermore, from the definitions of $F_{i}$ and $g_{i}$, we note that $\forall n \in N, g_{i}(n)$ is the position of the first 0 in the list $F_{i}(n-1, f(i \oplus n, 1)), F_{i}(n-2, f(i \oplus n, 2)), \cdots F_{i}\left(n-g_{i}(n), f\left(i \oplus n, g_{i}(n)\right)\right)$.

This means that $F_{i}\left(n-g_{i}(n), f\left(i \oplus n, g_{i}(n)\right)\right)=0$, but all preceding members of this sequence have a value of 1 .

\section{The Main Theorem}

Theorem 1 Suppose $f: N \times N \rightarrow N$ satisfies (1) $\exists t \in N$ such that $\forall n, x \in N, f(n, x)=$ $f(n+t, x)$ and (2) $\forall n, x \in N, f(n, x+1)-f(n, x) \geq-1$. Also, $\forall G_{i}, i=0,1,2, \cdots, t-1$, a base $B_{i}$ has been generated. Then the following is true,

1. $\forall i=0,1,2, \cdots, t-1, \forall b_{i k} \in B_{i}, g_{i}\left(b_{i k}\right)=b_{i k}$,

2. $\forall i=0,1,2, \cdots, t-1, \forall n \in N \backslash B_{i}, 1 \leq g_{i}(n)<n$,

3. $\forall i=0,1,2, \cdots, t-1, \forall n \in N \backslash B_{i}$,

(a) if $b_{i k}<n<b_{i, k+1}$ then $n=b_{i k}+\left(n-b_{i k}\right)$ and $g_{i}(n)=g_{i \oplus b_{i k}}\left(n-b_{i k}\right)$, and

(b) if $b_{i k}<n$ and $B_{i}$ is finite and $b_{i k}$ is the largest member of $B_{i}$, then $n=b_{i k}$ $+\left(n-b_{i k}\right)$ and $g_{i}(n)=g_{i \oplus b_{i k}}\left(n-b_{i k}\right)$.

Proof. We prove conclusions 1, 2, and 3 by mathematical induction on the pile size $n$. For each $n \in N$, we go through the same proof for each game $G_{i}, 0,1,2, \cdots, t-1$. So we can just focus our attention on any arbitrary $i=0,1,2, \cdots, t-1$. First, let $n=1$. Now $b_{i 0}=1 \in B_{i}$. Also, no matter what $f: N \times N \rightarrow N$ is, $g_{i}(1)=1$. Therefore, conclusion 1 holds for $n=1$. Conclusions 2, 3 do not apply to $n=1$.

Next, let $n=2$. Now $b_{i 1}=2 \in B_{i}$. Also, no matter what $f: N \times N \rightarrow N$ is, $g_{i}(2)=2$. Therefore, conclusion 1 holds for $n=2$. Conclusions 2,3 do not apply to 
$n=2$. So we can now use induction on $n$. In game $G_{i}$ let us suppose that conclusion 1 is true for all $b_{i j} \in\left\{b_{i_{0}}, b_{i_{1}}, \cdots, b_{i k}\right\}$ where $k \geq 1$ and conclusions 2,3 are true for all $n \in\left\{1,2,3,4,5, \cdots, b_{i k}\right\} \backslash B_{i}$. We show that conclusions 2, 3 are true for all $n \in\left\{b_{i k}+\right.$ $\left.1, b_{i k}+2, \cdots, b_{i, k+1}-1\right\}$ and conclusion 1 is true for $b_{i j}=b_{i, k+1}$. In the following argument, we omit the first part when $b_{i, k+1}-b_{i k}=1$. So we can imagine that $b_{i, k+1}-b_{i k} \geq 2$.

We will prove that conclusions 2,3 are true for $n \in\left\{b_{i k}+1, b_{i k}+2, \cdots, b_{i, k+1}-1\right\}$ by proving this sequentially with $n$ starting at $n=b_{i k}+1$ and ending at $n=b_{i, k+1}-1$.

Note that once we prove conclusion 3 for any $n \in\left\{b_{i k}+1, \cdots, b_{i, k+1}-1\right\}$, conclusion 2 will follow for this $n$ as well. This is because if $n=b_{i k}+\left(n-b_{i k}\right)$ where $1 \leq n-b_{i k}$ and $g_{i}(n)=g_{i \oplus b_{i k}}\left(n-b_{i k}\right)$, then $g_{i}(n)=g_{i \oplus b_{i k}}\left(n-b_{i k}\right) \leq n-b_{i k}<n$.

Recall that $g_{t}(m) \leq m$ is always true.

So let us now prove conclusion 3 -a is true for $n$ as $n$ varies sequentially over $b_{i k}+$ $1, b_{i k}+2, \cdots, b_{i, k+1}-1$. The proof for conclusion 3 -b is the same as 3 -a. Now since we are assuming that $b_{i, k+1}-b_{i k} \geq 2$, this means that $f\left(i \oplus b_{i k} \oplus 1,1\right)<b_{i k}$ is assumed as well.

This means that $f\left(i \oplus b_{i k} \oplus 1,1\right)=f\left(i \oplus\left(b_{i k}+1\right), 1\right)<g_{i}\left(\left(b_{i k}+1\right)-1\right)=g_{i}\left(b_{i k}\right)=b_{i k}$. By the definition of $g_{i}\left(b_{i k}+1\right)$, this implies $g_{i}\left(b_{i k}+1\right)=1$. Of course, $g_{i \oplus b_{i k}}(1)=1$. Therefore, $g_{i}\left(b_{i k}+1\right)=g_{i \oplus b_{i k}}(1)$. Therefore, suppose we have proved conclusion 3 for all $n \in\left\{b_{i k}+1, b_{i k}+2, \cdots, b_{i k}+t-1\right\}$ where $b_{i k}+t-1 \leq b_{i, k+1}-2$.

We now prove conclusion 3 for $n=b_{i k}+t$. This means we know that $g_{i}\left(b_{i k}+j\right)=$ $g_{i \oplus b_{i k}}(j), j=1,2,3, \cdots, t-1$ and we wish to prove $g_{i}\left(b_{i k}+t\right)=g_{i \oplus b_{i k}}(t)$.

Recall that $g_{i \oplus b_{i k}}(t)$ is the smallest positive integer $x$ such that the list

$$
\begin{aligned}
& \text { 1. } F_{i \oplus b_{i k}}\left(t-1, f\left(i \oplus b_{i k} \oplus t, 1\right)\right), F_{i \oplus b_{i k}}\left(t-2, f\left(i \oplus b_{i k} \oplus t, 2\right)\right), \ldots, \\
& \quad F_{i \oplus b_{i k}}\left(t-x, f\left(i \oplus b_{i k} \oplus t, x\right)\right)
\end{aligned}
$$

contains exactly one 0 (which comes at the end of the list). Also, $g_{i}\left(b_{i k}+t\right)$ is the smallest positive integer $x$ such that the list

$$
\text { 2. } \begin{aligned}
F_{i}\left(b_{i k}+t-1, f\left(i \oplus\left(b_{i k}+t\right), 1\right)\right), F_{i}\left(b_{i k}+t-2, f\left(i \oplus\left(b_{i k}+t\right), 2\right)\right), \ldots, \\
\\
F_{i}\left(b_{i k}+t-x, f\left(i \oplus\left(b_{i k}+t\right), x\right)\right)
\end{aligned}
$$

contains exactly one 0 (which comes at the end). Since we are assuming that $g_{i}\left(b_{i k}+\right.$ $j)=g_{i \oplus b_{i k}}(j), j=1,2, \cdots, t-1$, we know from the definition of $F_{i}$ and $F_{i \oplus b_{i k}}$ that $F_{i}\left(b_{i k}+j, y\right)=F_{i \oplus b_{i k}}(j, y)$ for all $j=1,2, \cdots, t-1$ and all $y \in N$. Recall that $F_{\theta}(n, x)=0$ when $1 \leq x \leq g_{\theta}(n)-1$ and $F_{\theta}(n, x)=1$ when $g_{\theta}(n) \leq x$.

Since $i \oplus b_{i k} \oplus t=i \oplus\left(b_{i k}+t\right)$, this means that lists (1) and (2) must be identical as long as $1 \leq x \leq t-1$. Now if $t \notin B_{i \oplus b_{i k}}$, we know by induction from conclusion 2 with game $G_{i \oplus b_{i k}}$ that $g_{i \oplus b_{i k}}(t)<t$. This tells us that for list (1) the smallest $x$ such that list (1) contains exactly one 0 satisfies $1 \leq x \leq t-1$. Therefore, since the two lists (1), (2) are identical when $1 \leq x \leq t-1$, this tells us that $g_{i}\left(b_{i k}+t\right)=g_{i \oplus b_{i k}}(t)$. 
Next, suppose $t \in B_{i \oplus b_{i k}}$. We know by induction from conclusion 1 with game $G_{i \oplus b_{i k}}$ that $g_{i \oplus b_{i k}}(t)=t$. Therefore, to prove $g_{i}\left(b_{i k}+t\right)=g_{i \oplus b_{i k}}(t)$ we need to show that $g_{i}\left(b_{i k}+t\right)=t$. Since $g_{i \oplus b_{i k}}(t)=t$, we know that the first $t-1$ members of the above list (1) consists of all 1's, and the $t^{\text {th }}$ member of list (1) is a 0 . Since the above lists (1) and (2) are identical when $1 \leq x \leq t-1$, we know that the first $t-1$ members of list (2) consists of all 1's. Therefore, to show that $g_{i}\left(b_{i k}+t\right)=t$, we need to show that the $t^{\text {th }}$ member of list (2) is a 0 .

From the definition of $b_{i, k+1}$, we know that $b_{i, k+1}-b_{i k}=b_{i \oplus b_{i k}, j}$ where $b_{i \oplus b_{i k}, j}$ is the smallest member of $B_{i \oplus b_{i k}}$ such that $f\left(i \oplus b_{i k} \oplus b_{i \oplus b_{i k}, j}, b_{i \oplus b_{i k}, j}\right) \geq b_{i k}$. Since $t \in B_{i \oplus b_{i k}}$ and $t<b_{i \oplus b_{i k}, j}$ we know that $f\left(i \oplus b_{i k} \oplus t, t\right)<b_{i k}=g_{i}\left(b_{i k}\right)$.

From this and $i \oplus b_{i k} \oplus t=i \oplus\left(b_{i k}+t\right)$ we know from the definition of $F_{i}$ that $F_{i}\left(b_{i k}+t-t, f\left(i \oplus\left(b_{i k}+t\right), t\right)\right)=F_{i}\left(b_{i k}, f\left(i \oplus b_{i k} \oplus t, t\right)\right)=0$, which means the $t^{t h}$ member of list (2) is a 0 .

This finishes conclusion 3. We now prove conclusion 1 for $b_{i, k+1}$. Therefore, we show that $g_{i}\left(b_{i, k+1}\right)=b_{i, k+1}$. Of course, $b_{i, k+1}=b_{i k}+b_{i \oplus b_{i k}, j}$ where $b_{i \oplus b_{i k}, j} \in B_{i \oplus b_{i k}}$ and $f\left(i \oplus b_{i k} \oplus b_{i \oplus b_{i k}, j}, b_{i \oplus b_{i k}, j}\right) \geq b_{i k}$. By induction with game $G_{i \oplus b_{i k}}, g_{i \oplus b_{i k}}\left(b_{i \oplus b_{i k}, j}\right)=b_{i \oplus b_{i k}, j}$. We now know that $g_{i}\left(b_{i k}+t\right)=g_{i \oplus b_{i k}}(t), t=1,2,3, \cdots, b_{i, k+1}-b_{i k}-1=b_{i \oplus b_{i k}, j}-1$.

Since $g_{i \oplus b_{i k}}\left(b_{i \oplus b_{i k}, j}\right)=b_{i \oplus b_{i k}, j}$, we know that all terms in the following list (3) are 1's, except the final term which is 0 .

$$
\text { 3. } \begin{aligned}
& F_{i \oplus b_{i k}}\left(b_{i \oplus b_{i k}, j}-1, f\left(i \oplus b_{i k} \oplus b_{i \oplus b_{i k}, j}, 1\right)\right), F_{i \oplus b_{i k}}\left(b_{i \oplus b_{i k}, j}-2, f\left(i \oplus b_{i k} \oplus b_{i \oplus b_{i k}, j}, 2\right)\right) \text {, } \\
& \quad \ldots, F_{i \oplus b_{i k}}\left(1, f\left(i \oplus b_{i k} \oplus b_{i \oplus b_{i k}, j}, b_{i \oplus b_{i k}, j}-1\right)\right), F_{i \oplus b_{i k}}\left(0, f\left(i \oplus b_{i k} \oplus b_{i \oplus b_{i k}, j}, b_{i \oplus b_{i k}, j}\right)\right)=0 .
\end{aligned}
$$

Now $g_{i}\left(b_{i, k+1}\right)=g_{i}\left(b_{i k}+b_{i \oplus b_{i k}, j}\right)$ is the position of the first 0 in list (4), where we note that the position of a term $F_{i}\left(b_{i k}+b_{i \oplus b_{i k}, j}-i, f\left(i \oplus\left(b_{i k}+b_{i \oplus b_{i k}, j}\right), i\right)\right)$ is $i$.

$$
\begin{aligned}
\text { 4. } & F_{i}\left(b_{i k}+b_{i \oplus b_{i k}, j}-1, f\left(i \oplus\left(b_{i k}+b_{i \oplus b_{i k}, j}\right), 1\right)\right), F_{i}\left(b_{i k}+b_{i \oplus b_{i k}, j}-2, f\left(i \oplus\left(b_{i k}+b_{i \oplus b_{i k}, j}\right), 2\right)\right), \\
& \ldots, F_{i}\left(b_{i k}+1, f\left(i \oplus\left(b_{i k}+b_{i \oplus b_{i k}, j}\right), b_{i \oplus b_{i k}, j}-1\right)\right), \\
& {\left[F_{i}\left(b_{i k}, f\left(i \oplus\left(b_{i k}+b_{i \oplus b_{i k}, j}\right), b_{i \oplus b_{i k}, j}\right)\right)\right]^{*} } \\
& F_{i}\left(b_{i k}-1, f\left(i \oplus\left(b_{i k}+b_{i \oplus b_{i k}, j}\right), b_{i \oplus b_{i k}, j}+1\right)\right), \\
& F_{i}\left(b_{i k}-2, f\left(i \oplus\left(b_{i k}+b_{i \oplus b_{i k}, j}\right), b_{i \oplus b_{i k}, j}+2\right)\right), \ldots, \\
& F_{i}\left(1, f\left(i \oplus\left(b_{i k}+b_{i \oplus b_{i k}, j}\right), b_{i \oplus b_{i k}, j}+b_{i k}-1\right)\right), \\
& F_{i}\left(0, f\left(i \oplus\left(b_{i k}+b_{i \oplus b_{i k}, j}\right), b_{i \oplus b_{i k}, j}+b_{i k}\right)\right)=0 .
\end{aligned}
$$

Since $g_{i}\left(b_{i k}+t\right)=g_{i \oplus b_{i k}}(t), t=1,2, \ldots, b_{i \oplus b_{i k}, j}-1$, and since $i \oplus\left(b_{i k}+b_{i \oplus b_{i k}, j}\right)=$ $i \oplus b_{i k} \oplus b_{i \oplus b_{i k}, j}$ as always we know from the definitions of $F_{i}$ and $F_{i \oplus b_{i k}}$ that the first $b_{i \oplus b_{i k}, j}-1$ terms of list (4) are the same as the first $b_{i \oplus b_{i k}, j}-1$ terms of list (3) which means that the first $b_{i \oplus b_{i k}, j}-1$ terms of list (4) are 1's.

Now $\left[F_{i}\left(b_{i k}, f\left(i \oplus\left(b_{i k}+b_{i \oplus b_{i k}, j}\right), b_{i \oplus b_{i k}, j}\right)\right)\right]^{*}=1$ from the definition of $F_{i}$ since $f\left(i \oplus\left(b_{i k} \oplus b_{i \oplus b_{i k}, j}\right), b_{i \oplus b_{i k}, j}\right) \geq g_{i}\left(b_{i k}\right)=b_{i k}$. 
Note that the last term in list (4) is 0 . As always, $\forall x \in N$ if $1 \leq x \leq b_{i k}-1$ then $g_{i}\left(b_{i k}-x\right) \leq b_{i k}-x$.

Also, from condition (2) on $f: N \times N \rightarrow N, f\left(i \oplus\left(b_{i k}+b_{i \oplus b_{i k}, j}\right), b_{i \oplus b_{i k}, j}+x\right) \geq$ $f\left(i \oplus\left(b_{i k}+b_{i \oplus b_{i k}, j}\right), b_{i \oplus b_{i k}, j}\right)-x \geq b_{i k}-x$.

Therefore, $\forall x \in N$ if $1 \leq x \leq b_{i k}-1$ then

$$
g_{i}\left(b_{i k}-x\right) \leq b_{i k}-x \leq f\left(i \oplus b_{i k} \oplus b_{i \oplus b_{i k}, j}, b_{i \oplus b_{i k}, j}+x\right) .
$$

From the definition of $F_{i}$, we now know that all terms in list (4) are 1's except the last term which is 0 . Therefore, since list (4) has $b_{i k}+b_{i \oplus b_{i k}, j}=b_{i, k+1}$ terms, we see that $g_{i}\left(b_{i, k+1}\right)=b_{i, k+1}$.

Bases for the $G_{i}, i=0,1,2, \ldots, t-1$. Suppose $f: N \times N \rightarrow N$ satisfies (1). $\exists t \in N$ such that $\forall n, x \in N, f(n, x)=f(n+t, x)$. For each $G_{i}, i=0,1,2, \ldots, t-1$, we assume that a base $B_{i}=\left(b_{i 0}=1, b_{i 1}, b_{i 2}, \ldots\right)$ and a function $\Theta_{i}: B_{i} \rightarrow N$ has been generated that satisfies the following conditions. First, $b_{i 0}=1, \Theta_{i}(1)=1, b_{i 1}=2, \Theta_{i}(2)=2$. Also, $\forall i \in\{0,1,2, \ldots, t-1\}, \forall k \geq 1, b_{i, k+1}=b_{i k}+b_{i \oplus b_{i k}, j}$ where $b_{i \oplus b_{i k}, j}$ is the smallest member of $B_{i \oplus b_{i k}}$ such that $\Theta_{i \oplus b_{i k}}\left(b_{i \oplus b_{i k}, j}\right)=b_{i \oplus b_{i k}, j}$ and $f\left(i \oplus b_{i k} \oplus b_{i \oplus b_{i k}, j}, b_{i \oplus b_{i k}, j}\right) \geq \Theta_{i}\left(b_{i k}\right)$ if such a $b_{i \oplus b_{i k}, j}$ exists. Also, once $b_{i \oplus b_{i k}, j}$ and $b_{i, k+1}$ are computed, we define $\Theta_{i}\left(b_{i, k+1}\right)=$ $\min \left\{b_{i \oplus b_{i k}, j}+\bar{x}: 1 \leq \bar{x} \leq b_{i k}\right.$ and $\left.f\left(i \oplus b_{i, k+1}, b_{i \oplus b_{i k}, j}+\bar{x}\right)<g_{i}\left(b_{i k}-\bar{x}\right)\right\}$.

Of course, $\min S$ is the smallest member of $S$. Also, each base $B_{i}$ has been generated as far as possible.

Theorem 2 Suppose $f: N \times N \rightarrow N$ satisfies (1) $\exists t \in N$ such that $\forall n, x \in N, f(n, x)=$ $f(n+t, x)$. Also, $\forall G_{i}, i=0,1,2, \ldots, t-1$, a base $B_{i}$ and a function $\Theta_{i}: B_{i} \rightarrow N$ have been generated.

Then the following is true.

1. $\forall i=0,1,2, \ldots, t-1, \forall b_{i k} \in B_{i}, g_{i}\left(b_{i k}\right)=\Theta_{i}\left(b_{i k}\right)$.

2. $\forall i=0,1,2, \ldots, t-1, \forall n \in N \backslash B_{i}, 1 \leq g_{i}(n)<n$.

3. $\forall i=0,1,2, \ldots, t-1, \forall n \in N \backslash B_{i}$

(a) if $b_{i k}<n<b_{i . k+1}$ then

$n=b_{i k}+\left(n-b_{i k}\right)$ and $g_{i}(n)=g_{i \oplus b_{i k}}\left(n-b_{i k}\right)$ and

(b) if $b_{i k}<n$ and $B_{i}$ is finite and $b_{i k}$ is the largest member of $B_{i}$, then $n=$ $b_{i k}+\left(n-b_{i k}\right)$ and $g_{i}(n)=g_{i \oplus b_{i k}}\left(n-b_{i k}\right)$.

Proof. The proof of Theorem 2 is very similar to the proof of Theorem 1 and is left to the reader. [5] gives a complete proof of Theorem 2 when $t=1$. Also, [5] gives several interesting applications of Theorem 2 for $t=1$. 
Remark As Theorem 1 shows, sometimes the bases for $G_{i}$ can be generated very easily. However, it is usually much harder to generate these bases. For these bases that are hard to generate, the reader might wonder what the value of the theory is. It turns out that quite often the members of $B_{i}$ grow exponentially. This means that even though the bases may be hard to generate very often they will give extremely efficient storage of the strategy of the game.

Example 2 Two alternating players play the game $G_{0}$ that uses the move function $f$ : $N \times N \rightarrow N$ defined by (1) $f(n, 3)=4$ if $n$ is odd and (2) $f(n, x)=2 x$ for all other $n, x \in N$. This means that $f(n, x)$ satisfies the conditions of Theorem 1 where $t=2$. This means that we must deal with the two games $G_{0}$ and $G_{1}$ and generate the two bases $B_{0}$ and $B_{1}$. This is reasonably easy to do. So we will just state the result and leave the details to the reader.

First, let $\left(F_{0}, F_{1}, F_{2}, F_{3}, \ldots\right)=(1,2,3,5,8,13,21, \ldots)$ denote the Fibonacci sequence. Then $B_{0}$ and $B_{1}$ are specified as follows where the pattern in braces [ ] repeats itself as we have illustrated.

$F_{0}, F_{1}, F_{2}, F_{3}, F_{4}, F_{5}$,
$B_{0}=\left\{\begin{array}{c}{\left[F_{6}+F_{1}, F_{7}+F_{2}+1, F_{8}+F_{3}+1, F_{9}+F_{4}, F_{10}+F_{5}-1, F_{11}+F_{6}-1\right],} \\ {\left[F_{12}+F_{7}, F_{13}+F_{8}+1, F_{14}+F_{9}+1, F_{15}+F_{10}, F_{16}+F_{11}-1, F_{17}+F_{12}-1\right], \ldots}\end{array}\right\}$
$B_{1}=\left\{\begin{array}{c}F_{0}, F_{1}, F_{2}, F_{3}, F_{4}+F_{1}, \\ F_{5}+F_{1},\left[F_{6}+F_{1}, F_{7}+F_{2}-1, F_{8}+F_{3}-1, F_{9}+F_{4}, F_{10}+F_{5}+1, F_{11}+F_{6}+1\right], \\ {\left[F_{12}+F_{7}, F_{13}+F_{8}-1, F_{14}+F_{9}-1, F_{15}+F_{10}, F_{16}+F_{11}+1, F_{17}+F_{12}+1\right], \ldots}\end{array}\right\}$

The reader may note that if $f(n, x)=2 x$, then $t=1$ and $B_{0}=\{1,2,3,5,8,13,21, \ldots\}$. This game is called Fibonacci Nim. In [3] we found all functions for which Theorem 1 is effective for $t=1$. Of course, $t=1$ when $f(n, x)=f(x)$.

\section{References}

[1] E. R. Berlekamp, J. H. Conway, and R. K. Guy, Winning Ways for Your Mathematical Plays, 2 (1982).

[2] C. L. Bouton, Nim, a game with a complete mathematical theory, Annals of Mathematics Princeton (2) 3 (1902), 35-39.

[3] Holshouser, A., J. Rudzinski, and H. Reiter, Dynamic One-Pile Nim, Fibonacci Quarterly, vol 41.3, June-July, 2003, pp 253-262.

[4] Flammenkamp, Achim, A. Holshouser, and H. Reiter, Dynamic One-Pile Blocking Nim, Electronic Journal of Combinatorics, Volume 10(1), 2003, \#N4.

[5] Holshouser, A., and H. Reiter, One Pile Nim with Arbitrary Move Function, Electronic Journal of Combinatorics, Volume 10(1), 2003, \#N7.

[6] Holshouser, A., and H. Reiter, Nimlike Games with Generalized Bases, Rocky Mountain Journal of Mathematics, to appear. 